\title{
Epidemiología de pacientes con Cáncer de Piel sometidos a Cirugía Micrográfica de Mohs en Solca-Guayaquil entre 2015 y
} 2017.

*Correspondencia:

carvajalmafernanda@gmail.com

Teléfono [593] 43718700 ext 2159

Conflicto de intereses: Los autores declaran no tener conflictos de intereses.

Fondos: Ver la página 101

Recibido: 1 Marzo 2018

Aceptado: 19 Agosto 2018

Publicado: 30 Agosto 2018

Membrete bibliográfico:

Carvajal M, Vásquez $M$. Epidemiología de pacientes con Cáncer de Piel sometidos a Cirugía Micrográfica de Mohs en SolcaGuayaquil entre 2015 y 2017 . Rev. Oncol. Ecu 2018;28(2):93-102.

DOI: https://doi.org/10.33821/269

Copyright Carvajal, et al. Este artículo es distribuido bajo los términos de Creative Commons Attribution License, el cual permite el uso y redistribución citando la fuente y al autor original.

\section{Epidemiology of Patients with Skin Cancer undergoing Mohs Micrographic Surgery at Solca-Guayaquill between 2015 and 2017.}

\section{María Fernanda Carvajal Ayala1* (D), María Lorena Vásquez².}

1. Instituto Oncológico Nacional "Dr. Juan Tanca Marengo", Solca - Guayaquil, Ecuador, Servicio de Dermatología - Piel y Partes Blandas.

2. Universidad Católica de Santiago de Guayaquil (UCSG), Guayaquil, Ecuador, postgrado de Cirugía Plástica Reconstructiva y Estética.

\section{Resumen}

Introducción: El cáncer de piel es la segunda neoplasia más común en la población guayaquileña, se presenta predominantemente en áreas expuestas a luz solar. Los tipos más frecuentes son el carcinoma basocelular y el escamocelular. La técnica de cirugía micrográfica de Mohs pese a ser descrita hace varias décadas continua vigente en el tratamiento de este tipo de neoplasias. El objetivo del estudio es presentar una serie de casos sometidos a esta cirugía.

Métodos: El presente estudio observacional, fue realizado en el Instituto Oncológico Nacional "Dr. Juan Tanca Marengo" Solca-Guayaquil. Se revisaron historias clínicas de los períodos comprendidos de enero del 2015 a diciembre del 2017. Se seleccionaron casos de pacientes con neoplasias malignas de la piel, sometidos a cirugía micrográfica de Mohs. El tipo de muestreo fue no probabilístico de conveniencia. Las variables estudiadas fuero sexo, edad, diagnóstico anatomopatológico, localización de las lesiones y recidivas. Se utiliza estadística descriptiva.

Resultados: Se analizaron 153 pacientes con edad media de $71.9 \pm 12$ años, el tipo histológico más frecuente fue el carcinoma basocelular nodular con 115 casos $(75.16 \%)$. Las lesiones se localizaron predominantemente en el dorso nasal 40 casos $(26.14 \%$ ) y en el ala nasal 17 casos $(11.11 \%)$. Al momento de la intervención quirúrgica más de la mitad de las lesiones se encontraban en estadio 1 (58.1\%). La técnica de cobertura empleada con mayor frecuencia fue el colgajo de rotación (49 \%). No se registraron casos de recidiva posterior a la cirugía micrográfica de Mohs en los pacientes estudiados. 
Conclusión: Las características epidemiológicas de cáncer de piel en la población de Guayaquil son similares a las reportadas en literatura internacional. Los pacientes sometidos a cirugía micrográfica de Mohs no presentaron recidiva en este reporte.

Palabras Claves: NEOPLASIAS CUTÁNEAS, /EPIDEMIOLOGÍA, CIRUGÍA DE MOHS.

DOI: $10.33821 / 269$

\section{Abstract}

Introduction: Skin cancer is the second most common neoplasm in the Guayaquil population. It occurs predominantly in areas exposed to sunlight. The most frequent types are basal cell carcinoma and squamous cell carcinoma. The technique of micrographic surgery of Mohs despite being described several decades ago continues in force in the treatment of this type of neoplasms. The aim of the study is to present a series of cases submitted to this surgery.

Methods: The present observational study was carried out in the National Oncological Institute "Dr. Juan Tanca Marengo "Solca-Guayaquil. Clinical histories of the periods between January 2015 and December 2017 were reviewed. Cases of patients with malignant neoplasms of the skin were selected, who underwent Mohs micrographic surgery. The type of sampling was non-probabilistic of convenience. The variables studied were sex, age, anatomopathological diagnosis, location of the lesions and recurrences. Descriptive statistics is used.

Results: 153 patients with a mean age of $71.9 \pm 12$ years were analyzed, the most frequent histological type was nodular basal cell carcinoma with 115 cases $(75.16 \%)$. The lesions were located predominantly on the nasal dorsum, 40 cases $(26.14 \%)$ and in the nasal ala, 17 cases $(11.11 \%)$. At the time of surgery, more than half of the lesions were in stage 1 (58.1\%). The coverage technique most frequently used was the rotation flap (49\%). There were no cases of recurrence after Mohs micrographic surgery in the patients studied.

Conclusion: The epidemiological characteristics of skin cancer in the population of Guayaquil are similar to those reported in international literature. Patients undergoing Mohs micrographic surgery did not present recurrence in this report.

Keywords: SKIN NEOPLASIAS, / EPIDEMIOLOGY, MOHS SURGERY.

DOI: $10.33821 / 269$

\section{Introducción}

El cáncer de piel es una enfermedad más común de lo que inicialmente se asume. Según datos del Registro de Tumores de Guayaquil en 2015 este tipo de neoplasia se encuentra en segundo lugar de frecuencia tanto en hombres como mujeres, siendo superados solo por los cánceres de próstata y mama respectivamente. Entre los cánceres de piel los de tipo no melanomas son los más frecuentes constituyendo el $90 \%$ de los tumores de la piel, de estos el $98 \%$ son principalmente carcinomas basocelulares y escamocelulares [1].

En Estados Unidos cada año se diagnostican cerca de 1.200.000 nuevos casos de cáncer no melanoma, de los cuales aproximadamente el $80 \%$ corresponde a carcinoma basocelular [2], esta neoplasia no presenta diferencias en presentación por sexo, 
presentándose con más frecuencia luego de los 40 años, es más común en caucásicos y está relacionado con la exposición a luz ultravioleta especialmente en quienes tienen piel tipo I y II que viven cerca de la línea ecuatorial; la exposición al sol puede actuar tanto en forma de daño acumulativo como por la exposición intermitente ocasionando daño en el ADN lo cual junto a la imposibilidad de la célula para repararlo desencadena en el desarrollo de carcinoma basocelular. Existen diversas variantes clínicas de carcinoma basocelular entre las cuales se encuentra nodular, superficial o multicéntrico, morfeiforme, quístico, infiltrativo, fibroepitelioma de Pinkus; de los cuales el tipo más común es el nodular [3,4].

Por otro lado, el carcinoma espinocelular es el segundo más frecuente luego del carcinoma basocelular, corresponde del 10 al $20 \%$ de todos los cánceres de piel, ocasiona la mayor cantidad de muertes en cáncer de piel no melanoma. Afecta principalmente a hombres mayores de 40 años con piel tipo I, guarda relación directa con la exposición intensa a luz del sol durante la vida, presentándose con más frecuencia en sitios expuestos a la luz como son cabeza, cuello y extremidades superiores [5].

Las alternativas de tratamiento para el cáncer de piel incluyen electrocoagulación y curetaje, laser de $\mathrm{CO} 2$, radioterapia, criocirugía, cirugía micrográfica de Mohs debiendo individualizar cada caso para la elección de la técnica más apropiada [6, 7].

Actualmente se considera a la cirugía de Mohs como el tratamiento ideal de varios tipos de cáncer de piel. Este procedimiento quirúrgico inicialmente fue introducido en la década de 1940, desarrollado por Frederic Edward Mohs en Wisconsin, Estados Unidos [8], esta se basa en el principio que los tumores cutáneos crecen de forma continua y se extiende por prolongaciones más allá del margen visible; en la técnica original se realizó fijación de tejido in vivo con una pasta de cloruro de zinc previo a removerlo y examinarlo al microscopio, debido al tiempo quirúrgico esta técnica careció de aceptación inmediata; sin embargo en 1974 Stegman y Tromovich reportaron que se podía omitir la fijación in situ sin presentar cambios en el resultado [9].

En la cirugía micrográfica de Mohs se realiza escisión de la lesión tumoral capa por capa, revisando sus márgenes y fondo quirúrgico al microscopio mediante congelación del tejido en fresco; en cada capa se diseña un mapa del tejido removido en caso de presentar tumor residual se procede a localizarlo en el mapa de forma que sirva de guía para en la siguiente capa solo remover material del área marcada con el fin de preservar la mayor cantidad de tejido posible a la par de extirpar todo el tumor. Las tinciones a utilizarse en el estudio microscópico incluyen hematoxilina eosina (carcinoma epidermoide), azul de toluidina (carcinoma basocelular) y técnicas de inmunohistoquímica para casos puntuales como CD 34 para dematofibrosarcoma protuberans [10]. Algo fundamental en este procedimiento quirúrgico es la comunicación entre el cirujano y el patólogo que realiza la revisión de los márgenes

Las principales indicaciones de la cirugía micrográfica de Mohs son tumores recurrentes, tumores localizados en zona $\mathrm{H}$ de la cara y zonas de alto riesgo de recurrencia, tamaño de tumor superior a $2 \mathrm{~cm}$ en cualquier localización excepto en la nariz donde la indicación es a partir de $1 \mathrm{~cm}$ [11]. El objetivo del presente estudio es presentar una serie de casos sometidos a esta cirugía en un centro oncológico en la ciudad de Guayaquil. 


\section{Materiales y Métodos}

El presente consiste en un estudio de tipo observacional, descriptivo, en un centro único desarrollado en el Instituto Oncológico Nacional "Dr. Juan Tanca Marengo" SolcaGuayaquil, en el cual se incluye datos de pacientes atendidos entre mes de enero del 2015 a diciembre de 2017. Ingresaron al estudio todos los pacientes con neoplasias malignas de la piel que fueron considerados para un tratamiento quirúrgico con cirugía micrográfica de Mohs. El tipo de muestreo fue no probabilístico de conveniencia. Se excluyeron casos en los que no se tuvo la información clínica completa. Previa autorización por los departamentos de investigación y estadística se recopilaron datos de las historias clínicas de los pacientes con cáncer de piel en quienes se realizó cirugía micrográfica de Mohs. Estos datos fueron ingresados en una matriz del software de análisis estadístico IBM SPSS 22. Las variables estudiadas fuero sexo, edad, diagnóstico anatomopatológico, localización de las lesiones. Se utiliza estadística descriptiva.

\section{Resultados}

Ingresaron al estudio 153 pacientes, 69 fueron hombres (45.1\%) y 84 mujeres (54.9\%).

El periodo con mayor cantidad de procedimientos micrográfico de Mohs correspondió al año 2016 con 68 procedimientos (44.4\%) como se aprecia en la Figura 1.

Figura 1. Distribución porcentual de procedimientos micrográficos de Mohs realizados en Solca entre 2015-2017.

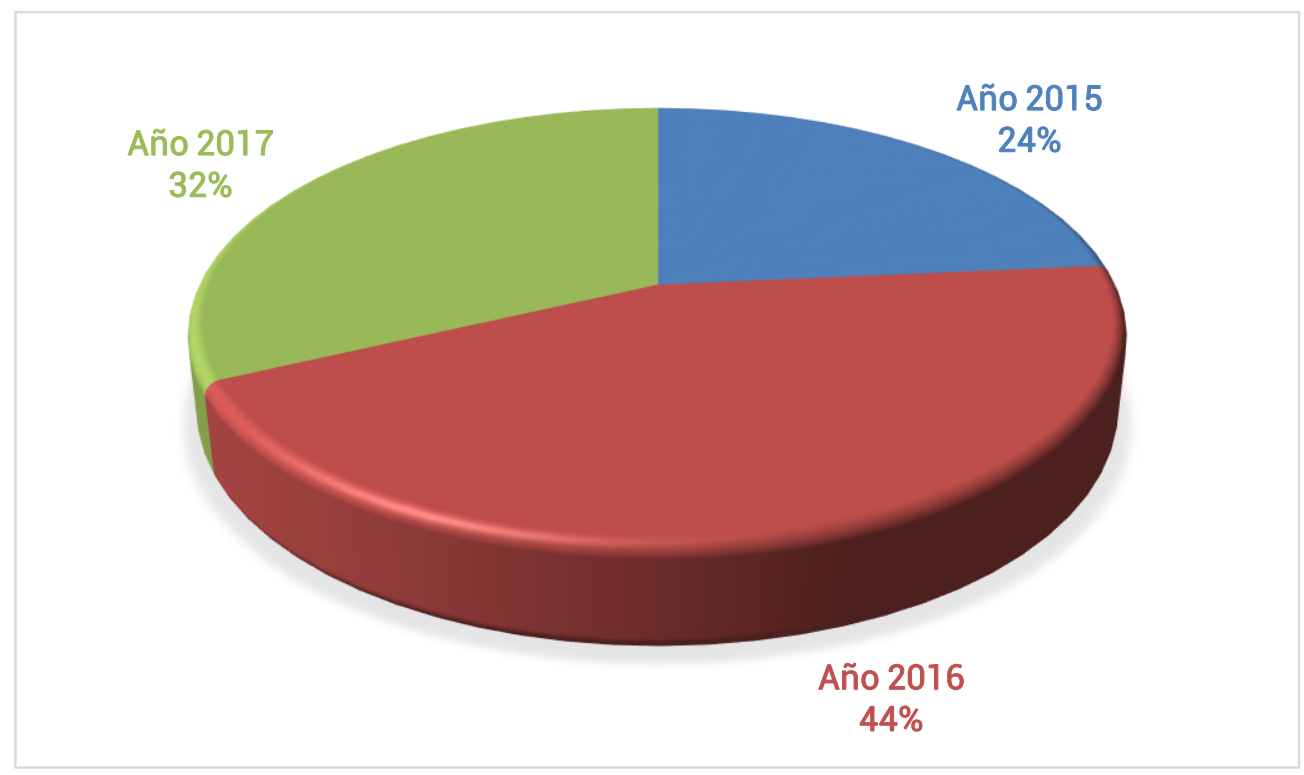


Con respecto a la edad de los pacientes en quienes se realizó cirugía micrográfica de Mohs, la edad minina es 42 años y la máxima 95 años; la media corresponde a 71.9 años con desviación estándar como medida de dispersión de 12.1 años; la mediana se ubica en 75 años al igual que la moda (Figura 2).

Figura 2. Histograma de la edad de los participantes al estudio.

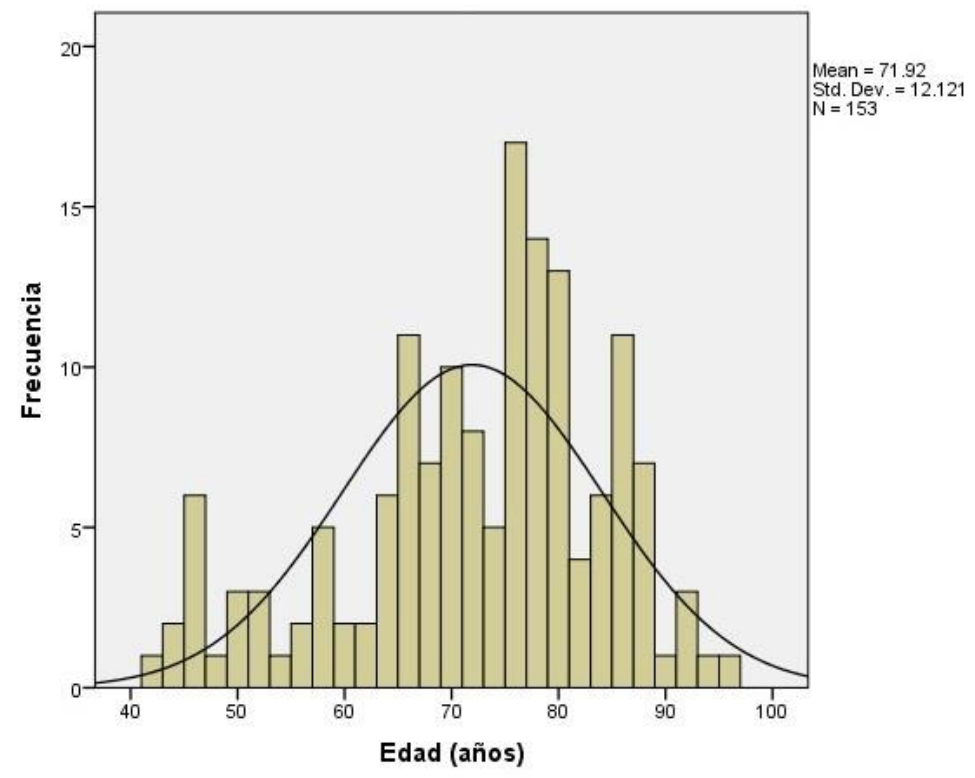

El análisis histológico de las lesiones reportó con mayor frecuencia Carcinoma basocelular nodular, al estar presente en el $75.16 \%$ de los casos $(n=115)$, seguido de carcinoma basocelular infiltrante con 11 casos $(7.19 \%)$ el resto de resultados se describen en la Tabla 1.

Tabla 1: Diagnóstico histológico de lesiones extraídas en cirugía micrográfica de Mohs.

\begin{tabular}{|l|l|l|}
\hline Diagnóstico & Frecuencia & Porcentaje \\
\hline Carcinoma basocelular nodular & 115 & $75.16 \%$ \\
\hline Carcinoma basocelular infiltrante & 11 & $7.19 \%$ \\
\hline Carcinoma escamocelular & 8 & $5.23 \%$ \\
\hline Carcinoma basocelular adenoide & 7 & $4.58 \%$ \\
\hline Carcinoma epidermoide & 3 & $1.96 \%$ \\
\hline Carcinoma escamocelular infiltrante & 3 & $1.96 \%$ \\
\hline Carcinoma basocelular pigmentado & 2 & $1.31 \%$ \\
\hline Carcinoma epidermoide ulcerado & 1 & $0.65 \%$ \\
\hline Carcinoma basocelular ulcerado & 1 & $0.65 \%$ \\
\hline Carcinoma basocelular bien diferenciado & 1 & $0.65 \%$ \\
\hline Carcinoma epidermoide infiltrante & 1 & $0.65 \%$ \\
\hline Total & 153 & $100 \%$ \\
\hline
\end{tabular}


La localización más frecuente de las lesiones fue el dorso nasal con una frecuencia de 40 (26.14\%), seguido del ala nasal (11.11\%) y de la región malar (8.5\%) en la Tabla 2 se muestra detalladamente las localizaciones de las lesiones extraídas en pacientes con cáncer de piel atendidos en el Hospital de Especialidades SOLCA de Guayaquil entre 2015 y 2017 a quienes se realizó cirugía micrográfica de Mohs.

Tabla 2. Localización de lesiones extraídas en cirugía micrográfica de Mohs en Solca entre 2015 y 2017.

\begin{tabular}{|l|l|l|}
\hline Tipo histológico & Frecuencia & Porcentaje \\
\hline Dorso nasal & 40 & $26.14 \%$ \\
\hline Ala nasal & 17 & $11.11 \%$ \\
\hline Región malar & 13 & $8.50 \%$ \\
\hline Punta nasal & 10 & $6.54 \%$ \\
\hline Canto interno & 8 & $5.23 \%$ \\
\hline Surco nasogeniano & 7 & $4.58 \%$ \\
\hline Región temporal & 6 & $3.92 \%$ \\
\hline Región frontal & 5 & $3.27 \%$ \\
\hline Region ciliar & 5 & $3.27 \%$ \\
\hline Ángulo mandibular & 4 & $2.61 \%$ \\
\hline Región preauricular & 4 & $2.61 \%$ \\
\hline Labio superior & 4 & $2.61 \%$ \\
\hline Canto externo & 4 & $2.61 \%$ \\
\hline Región interparietal & 3 & $1.96 \%$ \\
\hline Lóbulo de la oreja & 3 & $1.96 \%$ \\
\hline Mentón & 3 & $1.96 \%$ \\
\hline Pabellon auricular & 2 & $1.31 \%$ \\
\hline Brazo & 2 & $1.31 \%$ \\
\hline Entreceja & 2 & $1.31 \%$ \\
\hline Región palpebral & 2 & $1.31 \%$ \\
\hline Párpado inferior & 2 & $1.31 \%$ \\
\hline Región retroauricular & 2 & $1.31 \%$ \\
\hline Linea clavicular & 1 & $0.65 \%$ \\
\hline Muñeca & 1 & $0.65 \%$ \\
\hline Implantación del pelo frontal & 1 & $0.65 \%$ \\
\hline Región supraclavicular & 1 & $0.65 \%$ \\
\hline Labio inferior & 1 & $0.65 \%$ \\
\hline Total & 153 & $100.00 \%$ \\
\hline & & \\
\hline & 17 & \\
\hline & 17 & \\
\hline & 1 & \\
\hline
\end{tabular}

Al momento de la intervención quirúrgica más de la mitad de las lesiones se encontraban en estadio 1 (58.1\%). La figura 3 muestra la distribución porcentual de los diferentes estadios de lesión en la población estudiada. 
Figura 3. Estadios de lesión en pacientes a quienes se realizó procedimiento micrográfico de Mohs entre 2015 y 2017 en Solca-Guayaquil.

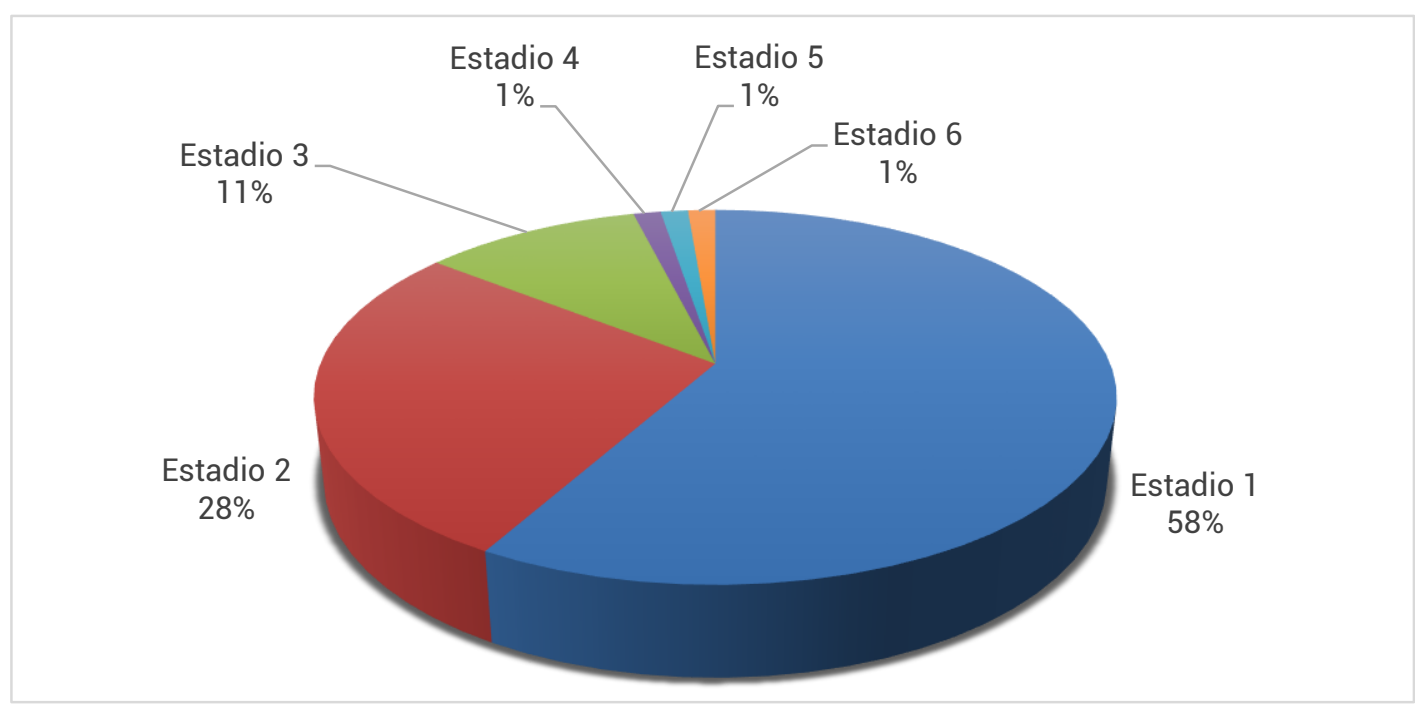

El colgajo de rotación fue la técnica de cobertura implementada con mayor frecuencia (49\%), la figura 4 muestra la distribución porcentual de las técnicas de cobertura utilizadas.

Figura 4. Técnicas de cobertura utilizadas en pacientes a quienes se realizó procedimiento micrográfico de Mohs entre 2015 y 2017 en SOLCA Guayaquil.

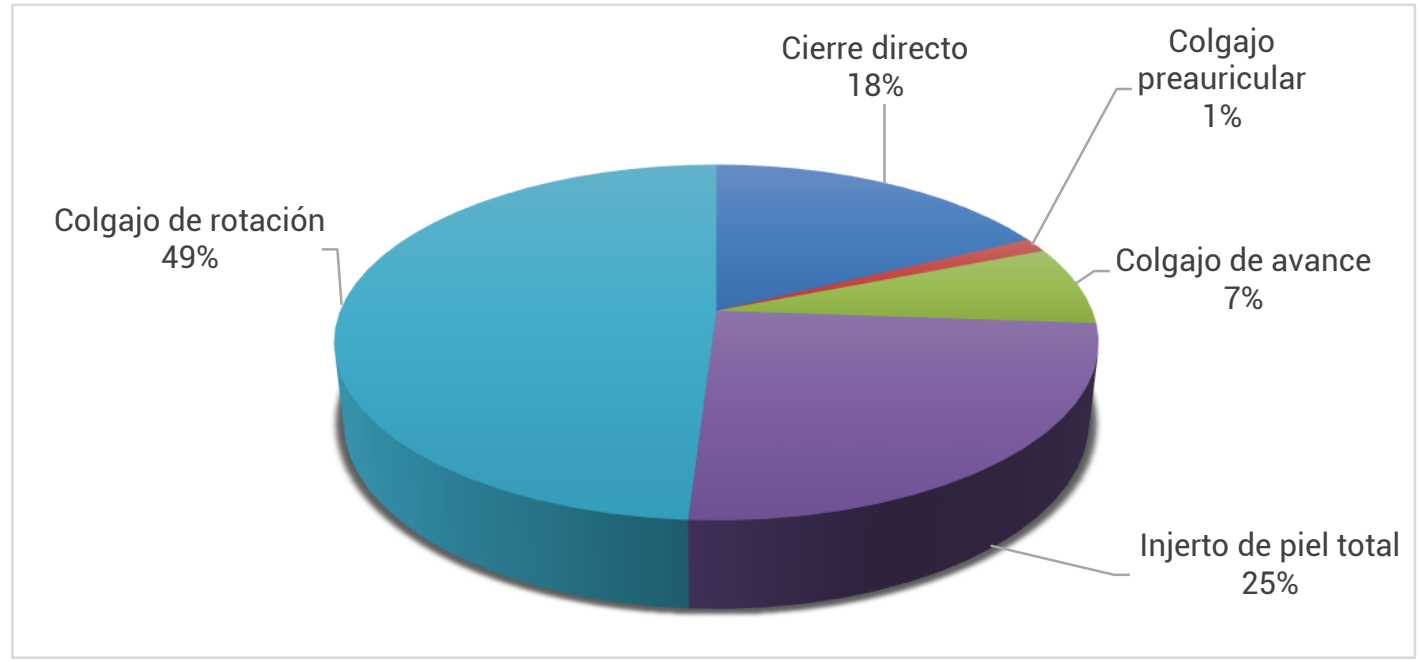

No se registraron casos de recidiva posterior a la cirugía micrográfica de Mohs en los pacientes estudiados. 


\section{Discusión}

El cáncer de piel constituye la segunda neoplasia maligna más común en la población de Guayaquil de ahí la importancia de conocer su epidemiologia, así como de realizar diagnóstico apropiado y oportuno, además de conocer las diferentes opciones terapéuticas disponibles en la actualidad [1].

En la población con cáncer de piel a quien se realizó cirugía micrográfica de Mohs en la Sociedad de Lucha Contra el Cáncer (SOLCA) entre 2015 y 2017, resaltaron los siguientes resultados: similitud en la distribución por sexo ( $54.9 \%$ y $45.1 \%$ a favor del sexo femenino). Edad media de 71.92 años presentado edad mínima de 42 y máxima de 95 años, lo cual coincide con lo descrito por Tilli et al y Breuninger et al quienes indican que el cáncer de piel presenta mayor incidencia luego de los 40 años [3, 4].

Durante el estudio histológico de las lesiones el $75.16 \%$ corresponde a carcinoma basocelular nodular y el $5.23 \%$ a carcinoma basocelular lo cual se aproxima a los datos reportados por Lobos et al en 2011 [2].

Todas las áreas donde se reportó la presencia de lesiones son áreas expuestas a luz solar y por tanto a rayos UV, lo cual guarda relación con el mecanismo fisiopatológico descrito en las publicaciones de Breuninger y Tilli $[3,4]$.

El colgajo de rotación fue la técnica de cobertura utilizada con mayor frecuencia, sin embargo es de aclarar que la elección de ésta depende del tamaño de la lesión así como la localización de la misma.

Pese a la existencia de diversas técnicas para el tratamiento del cáncer de piel como son electrocoagulación y curetaje, láser de $\mathrm{CO}_{2}$, radioterapia, criocirugía. La cirugía micrográfica de Mohs pese a tener varias décadas de haber sido creada, continua vigente siendo la técnica idónea para casos de cáncer de piel recurrentes o localizados en zonas de alto riesgo de recurrencia, lesiones superiores a $2 \mathrm{~cm}$ o lesiones mayores a $1 \mathrm{~cm}$ en la nariz [11].

\section{Conclusiones}

La cirugía micrográfica de Mohs pese a ser descrita hace más de 70 años continúa siendo una técnica vigente que da excelentes resultados en el tratamiento de cáncer de piel al confirmar la presencia de tejido libre de lesión mediante el estudio histopatológico. En la presente muestra no se presentaron casos de recidiva.

En la población estudiada se presentó cáncer de piel predominantemente en pacientes femeninos, la media para edad es 71.92 años el tipo celular más reportado es carcinoma basocelular nodular, localizándose con mayor frecuencia en el dorso de la nariz. 


\section{Agradecimientos}

Reconocemos a las personas que participaron indirectamente en el estudio tales como el personal técnico, pacientes y personal del Instituto Oncológico Nacional "Dr. Juan Tanca Marengo", Solca-Guayaquil.

\section{Información adicional}

Nota del Editor

La Revista Oncología Ecu permanece neutral con respecto a los reclamos jurisdiccionales en mapas publicados y afiliaciones institucionales.
Abreviaturas

ADN: Ácido Desoxiribonucleico.

$\mathrm{CO}_{2}$ : Dióxido de carbono.

UV: Ultravioleta.

\section{Archivos Adicionales}

Ninguno declarado por los autores.

\section{Fondos}

Los fondos de la investigación fueron propios de los autores del presente artículo.

\section{Disponibilidad de datos y materiales}

Existe la disponibilidad de datos bajo solicitud al autor de correspondencia. No se reportan otros materiales.

\section{Contribuciones de los autores}

MFC, LV, realizaron la idea de investigación, revisión bibliográfica. MFC, recolección de datos, escritura del artículo, análisis crítico del artículo. LV realizó las correcciones editoriales. Todos los autores leyeron y aprobaron la versión final del artículo.

\section{Aprobación de ética y consentimiento para participar}

No aplica a este estudio. 
No aplica.

\section{Información de los autores}

María Fernanda Carvajal Ayala, Médico Tratante del Servicio de Dermatología - Piel y Partes Blandas - Instituto Oncológico Nacional "Dr. Juan Tanca Marengo", Solca - Guayaquil, Ecuador. (iD https://orcid.org/0000-0002-3860-3400

María Lorena Vásquez, Posgradista de Cirugía Plástica Reconstructiva y Estética, Universidad Católica de Santiago de Guayaquil (UCSG), Guayaquil, Ecuador.

\section{Revisiones por pares}

Acceda a la revisión de pares académicos en el siguiente enlace: https://publons.com/review/4034563

\section{Referencias}

Abreviaturas en la referencias

DOI: Digital Object

Identifier

PMID: PubMed Identifier

SU: Short URL
1. Registro de Tumores SOLCA Matriz Guayaquil. Registro de Tumores SOLCA Matriz. [Online].; 2015 [cited 2018 Octubre 27. SU: goo.gl/jAThph

2. Lobos P, Lobos A. Cáncer de piel no - melanoma. Rev Med Clin Condes. 2011; 22(6): 737 - 748. DOI: 10.1016/S0716-8640(11)70486-2

3. Breuninger H, Sebastian G, Kortmann R. Brief guidelines: Basal cell carcinoma of the skin. J Dtsch Dermatol Ges. 2006; 4: 441 - 443.

4. Tilli C, Van Steensel M, Krekels G. Molecular etiology and pathogenesis of basal cell carcinoma. $\mathrm{Br} \mathrm{J}$ Der. 2005; 152: 1108-1124.

5. Buettner P, Raasch B. Incidence rates of skin cancer in Townswille, Australia. Int J Cancer. 1998; 78: 587 $-593$

6. Unlu R, Altun S, Kerme M. Is it really necessary to make wide excisions for basal cell carcinoma treatment? J Craniotac Surg. 2009; 20: 1989 - 1991.

7. Berlin J, Leeman D, Spunger J. Radiotherapy for rodentulcer type of basal cell carcinoma. Dermatol Surg. 2007; 33: 513-515.

8. Bobotsis R, Guenther L. How Mohs Surgery Transformed into a first-line Treatment of Skin Cancer. J Cutan Med Surg. 2017 Jan/Feb; 21(1): 40-41.

9. Tromovich T, Stegman S. Microscopically controlled excision of skin tumors. Arch Dermatol. 1974; 110: 231.

10. Thosani $M$, Marghoob A, Chen C. Current progress of inmmunostains in Mohs micrographic surgery: a review. Dermatol Surg. 2008; 34: 1621 - 1636.

11. Boeta Angeles L, Lacy Niebla RM. Cirugía micrográfica de Mohs. Dermatol Rev Mex. 2013; $57: 34$ - 40. 